\title{
Substantial Increase in the Incidence of Surgery for Osteoarthritis of the Base of the Thumb Between 1997 and 2019 in Finland
}

\author{
MERI MYOHANEN, EERO WARIS, PANU H. NORDBACK, SIMO MATTILA and SAMULI ASPINEN \\ Department of Hand Surgery, Helsinki University Hospital and University of Helsinki, Helsinki, Finland
}

\begin{abstract}
Background/Aim: Osteoarthritis (OA) at the trapeziometacarpal joint (TMC) is common. However, no published data on the TMC-OA surgery incidence exist. The aim of this study was to evaluate the incidence and trends of TMC-OA surgical treatment. Patients and Methods: A nationwide hospital discharge register-based study was conducted among patients surgically treated for TMC-OA in Finland between 1997 and 2019. Results: A total of 8,728 surgeries were performed. The annual surgery incidence increased 10-fold from 1997 to 2019. The incidence was higher in women and increased the most in the age groups of 50-59 and 60-69 years. TMC resection arthroplasty with or without ligament reconstruction and/or tendon interposition accounted for $91 \%$ of all surgeries. Conclusion: The increase in the surgical procedure rates is substantial. It is not explained by advancements in surgical treatment or by the ageing population. The indications for surgery appear to have become broader.
\end{abstract}

Osteoarthritis (OA) of the trapeziometacarpal joint (TMC) at the base of the thumb is one of the most common degenerative diseases. Female gender and heredity are known risk factors for hand OA (1). Moreover, the prevalence of TMC-OA increases with age (2-5). Up to $44 \%$ of Caucasian men and $57 \%$ of women $>60$ years of age have been reported to have signs of radiographic TMC-OA, whereas $3.7 \%$ of men and $9.3 \%$ of women suffered from symptomatic TMCOA leading to pain, weakness, and disability (5).

If non-operative measures fail to treat pain adequately in patients with TMC-OA, operative intervention is considered

This article is freely accessible online.

Correspondence to: Samuli Aspinen, Department of Hand Surgery, Helsinki University Hospital and University of Helsinki, Topeliuksenkatu 5 B, 00260 Helsinki, Finland. Tel: +35 8442847706,e-mail: samuli.aspinen@hus.fi

Key Words: Arthrodesis, arthroplasty, epidemiology, osteoarthritis.
(6). Trapeziectomy, described by Gervis in 1949 (7), is still the gold-standard surgical procedure for TMC-OA. Several different modifications of this procedure have been described, which combine e.g., ligament reconstruction, tendon interposition (LRTI), or both (8-10). Various interposition implants and total joint replacement designs have also been described (11). Arthrodesis is considered an option for patients with isolated TMC-OA (12).

While a major increase in hand-OA prevalence seems unlikely, a study by Wildin et al. showed that the number of patients seeing a surgeon for hand OA has almost tripled in 10 years from 13 to 34/100000 (13). In a Scandinavian study, more than 1 in 20 elderly women consulted a physician for TMC-OA (14). However, no national-level epidemiological studies on surgical treatment of TMC-OA have been previously reported. Thus, the purpose of this study was to investigate the incidence and trends of surgical treatment of TMC-OA in Finland between 1997 and 2019.

\section{Patients and Methods}

The Finnish National Hospital Discharge Register (FNHDR) is a statutory nationwide registry for all public and private hospitals. The database contains comprehensive information on patient characteristics, diagnoses, and surgical procedures performed in Finland. FNHDR data have been previously used in multiple epidemiologic studies $(15,16)$.

In this study, we analyzed FNHDR data of all surgical procedures on adults ( $>18$ years) for TMC-OA performed between 1 January 1997 to 31 December 2019. We investigated the number of surgical TMC-OA procedures using the 10th revision of the International Statistical Classification of Diseases and Related Health Problems (ICD-10) and the Finnish version of the Nordic Medico-Statistical Committee (NOMESCO) classifications of Surgical Procedures (NCSP). We included patients with ICD-10 diagnosis codes M15.0, M15.4, M15.8, M15.9, M18, M18.0, M18.1, M18.2, M18.3, M18.4, M18.5, M18.9, M19, M19.0, M19.1, M19.2, M19.8, and M19.9 as well as with NOMESCO procedure codes NDG60 (TMC arthroplasty), NDG70 (TMC arthrodesis), NDB58 (TMC joint replacement), and NDK00 (carpal bone incision or excision). Commonly, both complete or partial trapeziectomy with or without LRTI are performed under the code NDG60, but NDK00 may also 
be used for complete or partial trapeziectomy. Likewise, both interpositional and total joint replacement are performed under the code NDB58. Whereas NDG60, NDG70, and NDB58 are TMC joint-specific, NDK00 was only included when the corresponding diagnosis code was M18-M18.5, indicating TMC-OA.

The FNHDR data do not include information on the operated side or on the occurrence of revision procedures. Therefore, we only included the first procedure (primary procedure) of each patient in the final analysis of surgery incidence. The incidence of multiple procedures on the same patient are reported separately, indicating either operation of the contralateral hand or a revision of the hand primarily operated on.

To calculate the yearly incidence of each TMC-OA procedure in the population, we extracted annual data of the Finnish population, including the population distribution in 10-year age cohorts obtained from the public authority database Statistics Finland. Since the incidence of TMC-OA procedures was based on the actual surgery rates in the entire Finnish adult population, confidence intervals were not calculated. A statistical software program was used for analyzing entered data (IBM SPSS Statistics for Windows, Version 27.0. IBM Corp., Armonk, NY, USA).

Access to data was granted after Findata (data permit authority, operating at the National Institute for Health and Welfare) approved the study design. The data that support the findings of this study are available from Findata but restrictions apply to the availability of these data, which were used under license for the current study, and so are not publicly available. Data are however available from the authors upon reasonable request and with permission of Findata.

Ethical review and approval were waived for this study, due to the fact that it was a register-based analysis of blinded data. In such case, neither informed consent nor ethical approval are required according to Finnish legislation (medical research act 488/1999, amendments 295/2004, 794/2010).

\section{Results}

A total of 8,728 surgeries for TMC-OA were performed in Finland between 1997-2019, of which 7,256 (83\%) were primary procedures and included in further surgery incidence analysis. The total number of NDK00 procedures was 561, with 134 (24\%) cases linked to an M18-M18.5 diagnosis and thus included in the analysis. A total of 1,324 patients had two surgeries ( $83 \%$ females), 130 patients (95\% females) three, and 18 patients ( $78 \%$ females) had four surgeries, which was the maximum number of procedures performed on a single patient.

The number of all primary TMC-OA procedures increased 12-fold from 52 procedures in 1997 to 606 in 2019. The population of Finland was 5.16 million with an adult population of 3.99 million in 1997 and 5.53 million with an adult population of 4.48 million in 2019 . In relation to the adult population size, the incidence of TMC-OA surgery increased 10-fold from $1.3 / 100,000$ in 1997 to $13.5 / 100,000$ in 2019 . TMC arthroplasty (NDG60) was the most common surgical procedure, with a total of 6,095 procedures. This accounted for $84 \%$ of all primary procedures during the whole study period, with an increase in yearly surgery incidence from 49 to 469 procedures in 1997 and 2019, respectively. Moreover, 489 (6.7\%) carpal bone excisions (NDK00) were performed for TMC-OA, increasing the total amount of TMC resection arthroplasties with or without LRTI (NDG60+NDK00) to $91 \%$ during the study period. TMC arthrodeses (NDG70) and TMC joint replacements (NDB58) accounted for 541 (7.5\%) and 131 $(1.8 \%)$ of the cases (Figure 1).

The incidence of surgical treatment was higher among female patients; 5,789 (80\%) primary procedures were performed on women and 1,467 (20\%) on men. Out of the total number of primary procedures on female patients, 4,985 (86\%) were TMC arthroplasties (NDG60) and 320 (5.5\%) carpal bone excisions (NDK00), totaling the amount of TMC resection arthroplasties with or without LRTI (NDG60+NDK00) to 92\%. A total of 367 (6.3\%) TMC arthrodeses and $117(2.0 \%)$ TMC joint replacements were performed on female patients. Among male patients, TMC resection arthroplasties with or without LRTI (NDG60+NDK00) accumulated to 1,279 (87\%) procedures with 1,110 (76\%) TMC arthroplasties (NDG60) and 169 (12\%) carpal bone excisions (NDK00). TMC arthrodeses and TMC joint replacements comprised $174(12 \%)$ and 14 (1.0\%) operations, respectively (Table I).

The mean age of surgically treated TMC-OA patients was 58.9 years (8.4 SD, range $=20-96$ years) in women and 58.8 years (9.2 SD, range $=20-89$ years) in men. The most common age for surgical treatment among female patients in relation to the corresponding age group population was 5059 years, with 6.1 procedures/100,000 in 1997 and 59.2/100,000 in 2019 (Figure 2). Among male patients in 1997, TMC-OA procedures were most commonly performed on those 70-79 years, with an incidence of 2.3/100,000. In 2019, the age groups 50-59 and 60-69 showed equal-scale incidences of 12.7/100,000 and 14.4/100,000 (Figure 3).

\section{Discussion}

The nationwide population-based incidence of surgical treatment for TMC-OA in Finland increased 10-fold between 1997 and 2019. The increase in surgically treated TMC-OA was greatest in the age groups 50-59 and 60-69 years, i.e., among the working-age and early retirement-age population. The increase was significantly smaller among people $<50$ years of age, most likely owing to the lower incidence of TMC-OA in this age group. Elderly people generally have lower physical demands than their younger counterparts, which could explain the lower surgery rates in the 70-79 and $>80$ years age cohorts.

Consistent with the higher prevalence of TMC-OA in females, the incidence of primary TMC-OA surgery in this study was higher among women than men $(3,17)$. TMC resection arthroplasties with or without LRTI (NDG60+NDK00) accounted for most procedures, whereas 


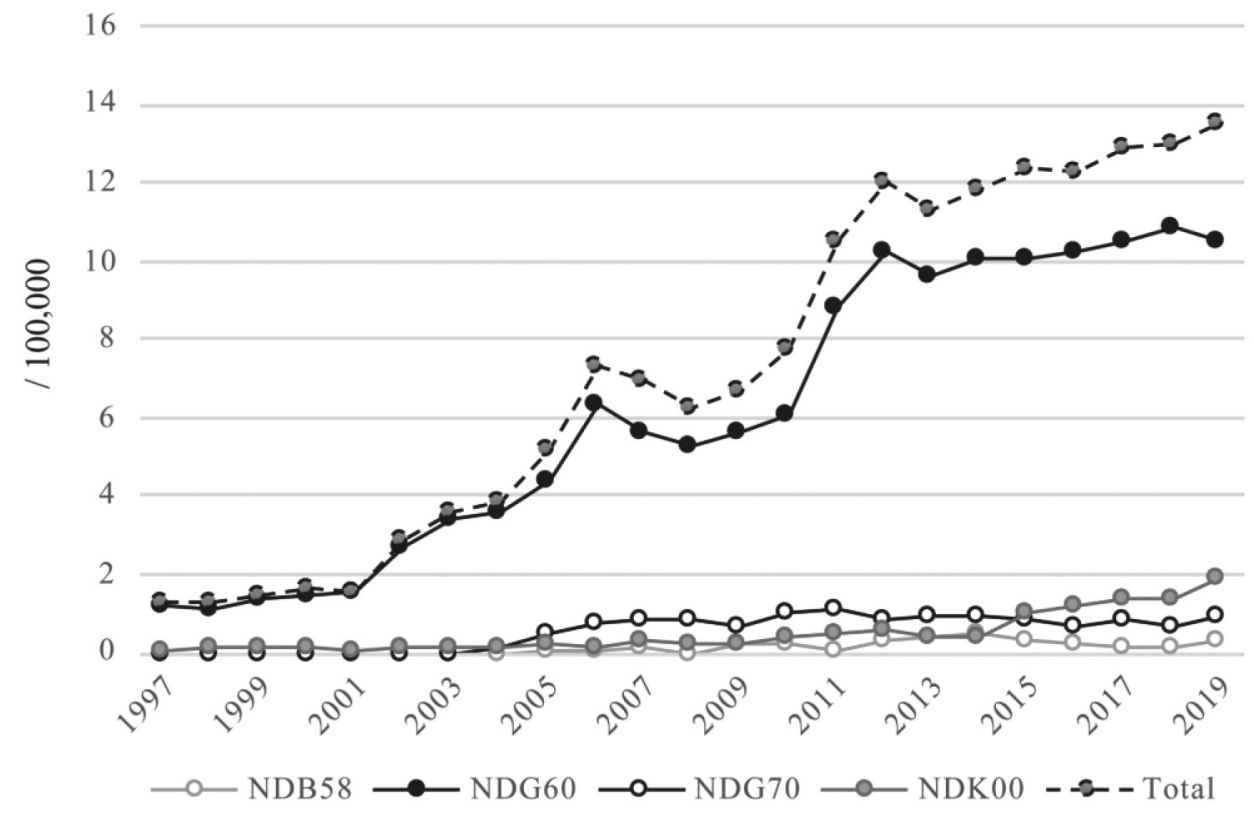

Figure 1. The yearly incidence of primary surgical trapeziometacarpal (TMC) osteoarthritis procedures in relation to the Finnish adult population per 100000 persons. NDB58: TMC joint replacement; NDG60: TMC arthroplasty; NDG70: TMC arthrodesis; and NDK00: carpal bone incision or excision. Only the first procedure for TMC-OA of each patient is included as primary surgical treatment.

the incidences of joint replacements and arthrodeses were relatively low, likely because these procedures do not address OA present at the scapho-trapezio-trapezoid (STT) joint, which becomes evident with disease progression. Moreover, higher risk for adverse events in TMC joint replacement and arthrodesis $(11,12)$ might explain the lower rates for these procedures. Noticeably, arthrodeses accounted for $12 \%$ of all surgeries performed on males, in contrast to $6.3 \%$ of all surgeries performed on females.

Only one previous study has investigated the incidence of TMC-OA surgical procedures. A Norwegian registry study from 1994 to 2011 covered 515 primary thumb TMC joint implant arthroplasties in 432 patients. Out of the 479 implant procedures included in the analysis, $371(76 \%)$ were due to OA. During the study period, the overall annual incidence of thumb TMC joint implant arthroplasties did not change. The number of joint implant arthroplasties performed for inflammatory arthritis decreased, whereas operations for OA increased (18). Our study yielded similar results with respect to an increase in TMC joint replacements, although their yearly number in Finland still remains small.

Similar to many other countries, there is an ongoing demographic ageing phenomenon in the Finnish population. In $1997,48 \%$ of the Finnish population was $\geq 40$ years, whereas in 2019 the proportion had increased to 54\%. The large post-World War II baby-boom generation, which in Finland is generally considered to be people born between $1945-1949$, is now in its 70s. With population ageing and
Table I. Number of all primary surgical procedures for trapeziometacarpal (TMC) osteoarthritis in Finland between 1997 and 2019 stratified by procedure type and sex.

\begin{tabular}{lccc}
\hline Procedure type & Total & Females & Males \\
\hline All primary procedures & 7,256 & $5,789(80 \%)$ & 1,467 \\
TMC arthroplasty (NDG60) & 6,095 & $4,985(82 \%)$ & 1,110 \\
TMC arthrodesis (NDG70) & 541 & $367(68 \%)$ & 174 \\
Carpal bone incision & 489 & $320(65 \%)$ & 169 \\
$\begin{array}{l}\text { or excision (NDK00) } \\
\text { TMC joint replacement (NDB58) }\end{array}$ & 131 & $117(89 \%)$ & 14 \\
\hline
\end{tabular}

since the prevalence of degenerative conditions, including TMC-OA, increases with age, it could be anticipated that the incidence of surgical interventions for degenerative conditions should increase accordingly. However, based on our results, the rising incidence of TMC-OA surgery is not explained by the ageing population, given the increase in surgery incidence across all age groups, even when adjusted to corresponding age group population data.

No advances in surgical techniques have been developed for TMC-OA that could explain the increase in surgery incidence during the study period. In contrast, in recent years there has been a shift back towards simple trapeziectomy along with accumulating evidence of its non-inferiority compared to trapeziectomy combined with LRTI procedures $(19,20)$ and the higher failure rates of implant procedures 


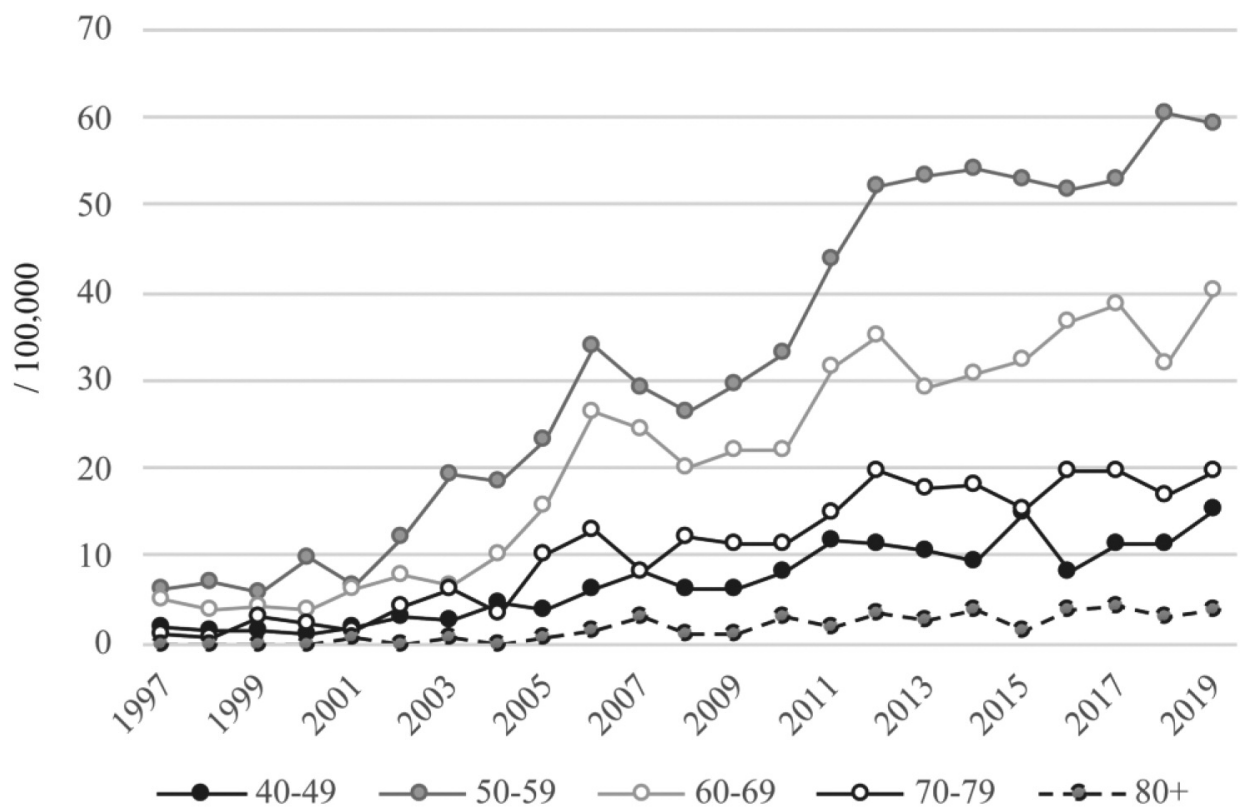

Figure 2. The yearly incidence of primary surgical trapeziometacarpal osteoarthritis (TMC-OA) treatment in females. The incidence is reported in 10-year age cohorts per 100,000 persons in the corresponding age group population. Only the first procedure for TMC-OA of each patient is included as primary surgical treatment.

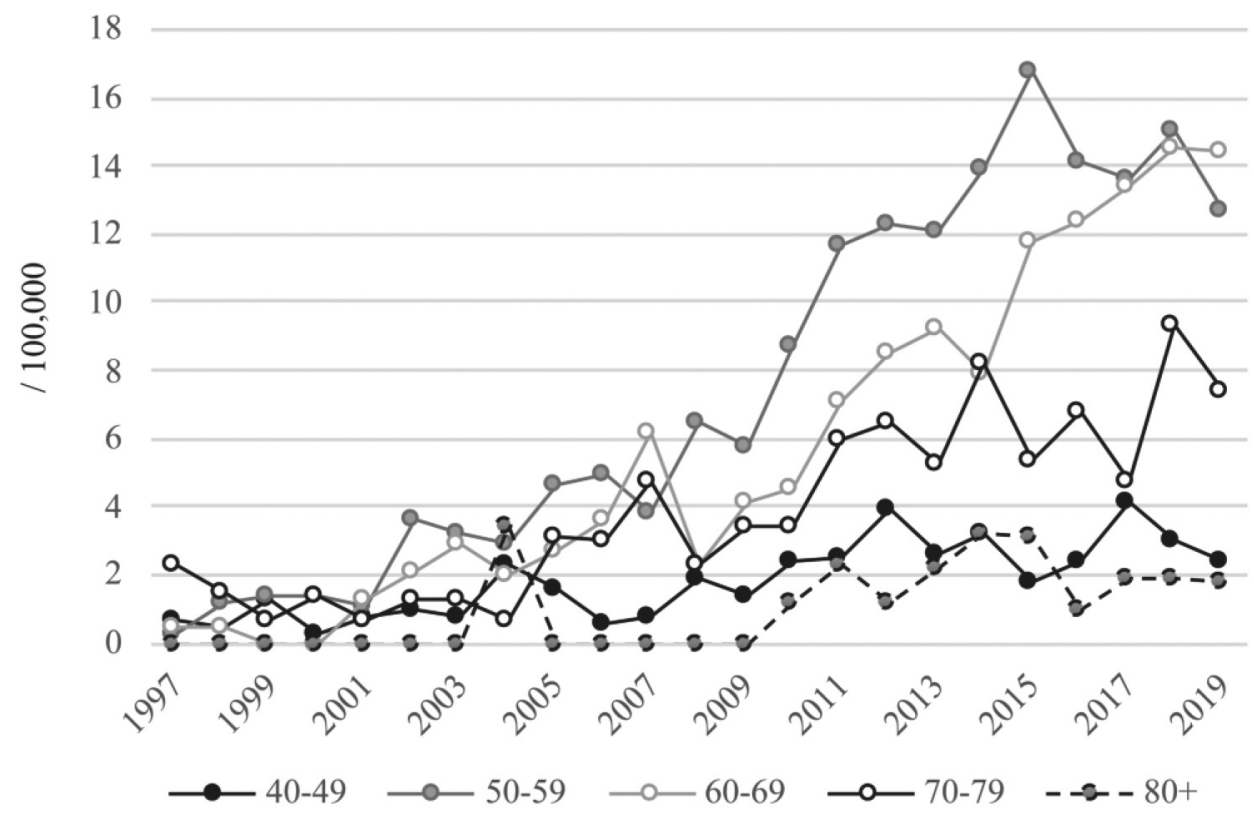

Figure 3. The yearly incidence of primary surgical trapeziometacarpal osteoarthritis (TMC-OA) treatment in males. The incidence is reported in 10-year age cohorts per 100,000 persons in the corresponding age group population. Only the first procedure for TMC-OA of each patient is included as primary surgical treatment.

(11). This is also consistent with our findings of persistently low rates of joint replacement in Finland. Regardless of the absence of recent advancements in surgical techniques, the results of TMC resection arthroplasties with or without LRTI are known to be good in terms of pain management and patient satisfaction based on observational studies $(21,22)$, which could be the underlying reason for the rising popularity of TMC surgical procedures. 
The natural progression of TMC-OA symptoms is still poorly known and the impact of physical, functional, and psychological factors on clinical manifestations of TMC-OA remain unidentified. Moreover, long-term outcomes after non-operative treatment of painful TMC-OA have not been reported (23). Low quality studies suggest that hand exercise may have a mild positive effect on hand pain, function and finger joint stiffness in the short term (23), however, an occupational therapy regimen has not been shown to significantly delay or reduce the rate of surgery (24). As a growing number of patients, even with few or mild symptoms, are seeking expert opinion on TMC-OA treatment $(13,14,25)$, high-quality research is warranted to aid decision making between non-operative and operative treatment in order to avoid surgical over-treatment.

The increasing incidence of TMC-OA surgery is consistent with previous Scandinavian studies for other degenerative conditions. For example, lumbar decompressions and spine fusions increased by 155\% in Finland between 1997 and 2018 (26). Another Finnish study reported that the incidence of hip arthroplasty for primary OA in patients 30-59 years increased 6-fold between 1980 and 2007 (27). Moreover, the incidence of knee arthroplasty increased 2.1-fold in Finland between 2000 and 2012, 6.0-fold in Denmark, 3.9-fold in Norway, and 2.3-fold in Sweden between 1997 and 2012 (28).

The strength of our study is the high-quality nationwide nature of the data. The FNHDR is mandatory for all Finnish public and private hospitals. The representativeness of the register has shown to be excellent for the last two decades. Recent studies on pertrochanteric fractures and cruciate ligament injuries reported a FNHDR coverage of 92-98\% and accuracy of $88-89 \%$ in procedural coding $(15,16)$.

There are some limitations to the FNHDR data. First, there is a possibility of missing defectively coded diagnoses/procedures (e.g., NDK00 procedures for TMC-OA combined with M19.X diagnoses). Second, because both simple trapeziectomy and trapeziectomy with LRTI are performed under the same procedural codes (NDG60 and NDK00), it is impossible to report the current trend between these two most common procedures. Likewise, it is impossible to separate interpositional and total joint replacement (NDB58). Third, the side of the operation or possible reoperations on the same extremity are not distinctly recorded in the database. Therefore, we excluded all but the first procedure of each patient in the incidence analysis. Due to this limitation, some revision procedures with the primary operation performed before 1997 might be included in the incidence analysis. However, we expect this number to be minimal due to the low operation rates during the early years of this study and the low revision rate for TMC resection arthroplasties with or without LRTI $(4.6 \%)$ reported in our previous study (29). Likewise, we may assume that most of the 1,176 second operations to be bilaterally operated patients rather than revisions. Only 148 patients were operated three or more times for TMC-OA in this study. Another limitation of our study is the lack of data concerning possible changes in the prevalence of TMC-OA in Finland during the study period. The prevalence of hip and knee OA has remained unchanged in Finland over the last decades (30) and we consider the probability of major changes in TMC-OA prevalence to be low.

Considering the drastic rise in the incidence of surgery for TMC-OA in Finland, cautious assumptions may be made of either surgical undertreatment in the early years of the study, overtreatment in the later years, or both. As the rising surgery incidence observed in this study is not explained by changing population demographics, TMC-OA prevalence, or advances in surgical techniques, the indications for surgery of TMC-OA appear to have become broader.

\section{Conflicts of Interest}

The Authors declare that they have no competing interests in relation to this study.

\section{Authors' Contributions}

S.A. conceived the study. All authors contributed to designing the study. S.A. and E.W. acquired the data. S.A. and M.M. performed the data analysis and M.M. drafted the manuscript. All Authors contributed to refinement and approved the final manuscript.

\section{References}

1 Kalichman L and Hernández-Molina G: Hand osteoarthritis: an epidemiological perspective. Semin Arthritis Rheum 39(6): 465476, 2010. PMID: 19482338. DOI: 10.1016/j.semarthrit. 2009.03.001

2 Dahaghin S, Bierma-Zeinstra SM, Ginai AZ, Pols HA, Hazes JM and Koes BW: Prevalence and pattern of radiographic hand osteoarthritis and association with pain and disability (the Rotterdam study). Ann Rheum Dis 64(5): 682-687, 2005. PMID: 15374852. DOI: 10.1136/ard.2004.023564

3 Haara MM, Heliövaara M, Kröger H, Arokoski JP, Manninen P, Kärkkäinen A, Knekt $\mathrm{P}$, Impivaara $\mathrm{O}$ and Aromaa $\mathrm{A}$ : Osteoarthritis in the carpometacarpal joint of the thumb. Prevalence and associations with disability and mortality. J Bone Joint Surg Am 86(7): 1452-1457, 2004. PMID: 15252092. DOI: 10.2106/00004623-200407000-00013

4 Wilder FV, Barrett JP and Farina EJ: Joint-specific prevalence of osteoarthritis of the hand. Osteoarthritis Cartilage 14(9): 953957, 2006. PMID: 16759885. DOI: 10.1016/j.joca.2006.04.013

5 Zhang Y, Xu L, Nevitt MC, Niu J, Goggins JP, Aliabadi P, Yu W, Lui LY and Felson DT: Lower prevalence of hand osteoarthritis among Chinese subjects in Beijing compared with white subjects in the United States: the Beijing Osteoarthritis Study. Arthritis Rheum 48(4): 1034-1040, 2003. PMID: 12687546. DOI: $10.1002 /$ art. 10928

6 Kloppenburg M, Kroon FP, Blanco FJ, Doherty M, Dziedzic KS, Greibrokk E, Haugen IK, Herrero-Beaumont G, Jonsson H, 
Kjeken I, Maheu E, Ramonda R, Ritt MJ, Smeets W, Smolen JS, Stamm TA, Szekanecz Z, Wittoek R and Carmona L: 2018 update of the EULAR recommendations for the management of hand osteoarthritis. Ann Rheum Dis 78(1): 16-24, 2019. PMID: 30154087. DOI: 10.1136/annrheumdis-2018-213826

7 Gervis WH: Excision of the trapezium for osteoarthritis of the trapezio-metacarpal joint. J Bone Joint Surg Br 31B(4): 537-9, illust, 1949. PMID: 15397137.

8 Burton RI and Pellegrini VD Jr: Surgical management of basal joint arthritis of the thumb. Part II. Ligament reconstruction with tendon interposition arthroplasty. J Hand Surg Am 11(3): 324-332, 1986. PMID: 3711604. DOI: 10.1016/s0363-5023(86)80137-x

9 Kaarela $\mathrm{O}$ and Raatikainen T: Abductor pollicis longus tendon interposition arthroplasty for carpometacarpal osteoarthritis of the thumb. J Hand Surg Am 24(3): 469-475, 1999. PMID: 10357523. DOI: $10.1053 /$ jhsu.1999.0469

10 Weilby A: Tendon interposition arthroplasty of the first carpometacarpal joint. J Hand Surg Br 13(4): 421-425, 1988. PMID: 3249143. DOI: 10.1016/0266-7681(88)90171-4

11 Ganhewa AD, Wu R, Chae MP, Tobin V, Miller GS, Smith JA, Rozen WM and Hunter-Smith DJ: Failure rates of base of thumb arthritis surgery: a systematic review. J Hand Surg Am 44(9): 728741 .e10, 2019. PMID: 31262534. DOI: 10.1016/j.jhsa.2019.05.003

12 Vermeulen GM, Brink SM, Slijper H, Feitz R, Moojen TM, Hovius SE and Selles RW: Trapeziometacarpal arthrodesis or trapeziectomy with ligament reconstruction in primary trapeziometacarpal osteoarthritis: a randomized controlled trial. J Bone Joint Surg Am 96(9): 726-733, 2014. PMID: 24806009. DOI: 10.2106/JBJS.L.01344

13 Wildin C, Dias JJ, Heras-Palou C, Bradley MJ and Burke FD: Trends in elective hand surgery referrals from primary care. Ann R Coll Surg Engl 88(6): 543-546, 2006. PMID: 17059713. DOI: 10.1308/003588406X117070

14 Moriatis Wolf $\mathrm{J}$, Turkiewicz A, Atroshi I and Englund M: Prevalence of doctor-diagnosed thumb carpometacarpal joint osteoarthritis: an analysis of Swedish health care. Arthritis Care Res (Hoboken) 66(6): 961-965, 2014. PMID: 24339432. DOI: $10.1002 /$ acr. 22250

15 Huttunen TT, Kannus P, Pihlajamäki H and Mattila VM: Pertrochanteric fracture of the femur in the Finnish National Hospital Discharge Register: validity of procedural coding, external cause for injury and diagnosis. BMC Musculoskelet Disord 15: 98, 2014. PMID: 24655318. DOI: 10.1186/1471-2474-15-98

16 Mattila VM, Sillanpää P, Iivonen T, Parkkari J, Kannus P and Pihlajamäki H: Coverage and accuracy of diagnosis of cruciate ligament injury in the Finnish National Hospital Discharge Register. Injury 39(12): 1373-1376, 2008. PMID: 18703187. DOI: 10.1016/j.injury.2008.05.007

17 Zhang Y, Niu J, Kelly-Hayes M, Chaisson CE, Aliabadi P and Felson DT: Prevalence of symptomatic hand osteoarthritis and its impact on functional status among the elderly: The Framingham Study. Am J Epidemiol 156(11): 1021-1027, 2002. PMID: 12446258. DOI: 10.1093/aje/kwf141

18 Krukhaug Y, Lie SA, Havelin LI, Furnes O, Hove LM and Hallan G: The results of 479 thumb carpometacarpal joint replacements reported in the Norwegian Arthroplasty Register. J Hand Surg Eur Vol 39(8): 819-825, 2014. PMID: 24784114 DOI: $10.1177 / 1753193413513988$

19 Li YK, White C, Ignacy TA and Thoma A: Comparison of trapeziectomy and trapeziectomy with ligament reconstruction and tendon interposition: a systematic literature review. Plast Reconstr Surg 128(1): 199-207, 2011. PMID: 21399560. DOI: 10.1097/PRS.0b013e318217435a

20 Wajon A, Vinycomb T, Carr E, Edmunds I and Ada L: Surgery for thumb (trapeziometacarpal joint) osteoarthritis. Cochrane Database Syst Rev (2): CD004631, 2015. PMID: 25702783. DOI: 10.1002/14651858.CD004631.pub4

21 Gangopadhyay S, McKenna H, Burke FD and Davis TR: Fiveto 18-year follow-up for treatment of trapeziometacarpal osteoarthritis: a prospective comparison of excision, tendon interposition, and ligament reconstruction and tendon interposition. J Hand Surg Am 37(3): 411-417, 2012. PMID: 22305824. DOI: $10.1016 /$ j.jhsa.2011.11.027

22 Salem H and Davis TR: Six year outcome excision of the trapezium for trapeziometacarpal joint osteoarthritis: is it improved by ligament reconstruction and temporary Kirschner wire insertion? J Hand Surg Eur Vol 37(3): 211-219, 2012. PMID: 22025011. DOI: 10.1177/1753193411414516

23 Østerås N, Kjeken I, Smedslund G, Moe RH, SlatkowskyChristensen B, Uhlig $\mathrm{T}$ and Hagen KB: Exercise for hand osteoarthritis. Cochrane Database Syst Rev 1: CD010388, 2017. PMID: 28141914. DOI: 10.1002/14651858.CD010388.pub2

24 Gravås EMH, Østerås N, Nossum R, Eide REM, Klokkeide Å, Matre KH, Olsen M, Andreassen O, Haugen IK, Tveter AT and Kjeken I: Does occupational therapy delay or reduce the proportion of patients that receives thumb carpometacarpal joint surgery? A multicentre randomised controlled trial. RMD Open 5(2): e001046, 2019. PMID: 31798953. DOI: 10.1136/rmdopen2019-001046

25 Gravås EMH, Tveter AT, Nossum R, Eide REM, Klokkeide A, Matre KH, Olsen M, Andreassen Ø, Østerås N, Haugen IK and Kjeken I: Non-pharmacological treatment gap preceding surgical consultation in thumb carpometacarpal osteoarthritis - a crosssectional study. BMC Musculoskelet Disord 20(1): 180, 2019. PMID: 31039774. DOI: 10.1186/s12891-019-2567-3

26 Ponkilainen VT, Huttunen TT, Neva MH, Pekkanen L, Repo JP and Mattila VM: National trends in lumbar spine decompression and fusion surgery in Finland, 1997-2018. Acta Orthop 92(2): 199-203, 2021. PMID: 33106074. DOI: 10.1080/17453674.2020.1839244

27 Skyttä ET, Jarkko L, Antti E, Huhtala H and Ville R: Increasing incidence of hip arthroplasty for primary osteoarthritis in 30- to 59-year-old patients. Acta Orthop 82(1): 1-5, 2011. PMID: 21189098. DOI: 10.3109/17453674.2010.548029

28 NiemeläInen MJ, MäKelä KT, Robertsson O, W-Dahl A, Furnes O, Fenstad AM, Pedersen AB, Schrøder HM, Huhtala H and Eskelinen A: Different incidences of knee arthroplasty in the Nordic countries. Acta Orthop 88(2): 173-178, 2017. PMID: 28056570. DOI: $10.1080 / 17453674.2016 .1275200$

29 Mattila S and Waris E: Revision of trapeziometacarpal arthroplasty: risk factors, procedures and outcomes. Acta Orthop 90(4): 389-393, 2019. PMID: 30931687. DOI: 10.1080/ 17453674.2019.1599253

30 Arokoski JPA, Manninen P, Kröger H, Heliövaara M, Nykyri E and Impivaara O: Changes in morbidity. In: Musculoskeletal disorders and issues in Finland. Helsinki, Publications of the National Public Health Institute, pp. 60-61, 2007.

Received January 3, 2022

Revised January 17, 2022

Accepted January 20, 2022 